\title{
Importancia De La Innovación Para Emprendedores De Micro Y Pequeñas Empresas Mexicanas Del Sector Servicio En Villahermosa-Tabasco Y Mexicali-Baja California. Estudio Cuantitativo
}

\author{
Jahicela Liévano, Doctorante \\ Paulina Vargas, Doctorante \\ Beatriz Pico, Dra. \\ Juan Pérez, Dr.
}

Planeación Estratégica y Dirección de Tecnologías, UPAEP, México

Doi:10.19044/esj.2018.v14n22p304 URL:http://dx.doi.org/10.19044/esj.2018.v14n22p304

\begin{abstract}
The enterprises that result in the creation of companies are in need of implementing strategies that translate into an adequate positioning of their sector against competition. It also allows them to survive in global markets and to undergo continuous changes. For this reason, innovation as a part of the project is an important differentiating factor. In this context, this paper focuses on contrasting the actions focused on innovation by entrepreneurs of micro and small Mexican companies in the service sector of Mexicali, Baja California, and Villahermosa, Tabasco, with the aim of identifying the significant role played by innovation in the course of time. The above was achieved through a quantitative study that has a descriptive, exploratory, and correlational scope. As part of the results, it is through these strategies that it helps to promote an innovative enterprise, facilitating the generation of new ideas, and improving its ability to innovate. This would, however, results to products and services that are scalable and of greater value, taking the company to competitive levels and promoting its development in a sustainable manner. In conclusion, this effort must be permanent because there is a positive and significant relationship between innovation and actions in the development of it. This is not only for the company, but for the entity where they are located.
\end{abstract}

Keywords: entrepreneurship, innovation, micro and small businesses 


\section{Resumen}

Los emprendimientos que resultan en la creación de empresas se ven en la necesidad de implementar estrategias que se traduzcan en un posicionamiento adecuado en su sector frente a su competencia y que además les permita sobrevivir en mercados globales y de continuos cambios. Por esta razón, la innovación como parte de los emprendimientos resulta un factor diferenciador importante. Bajo este contexto, la investigación tuvo como objetivo contrastar las acciones enfocadas a la innovación por parte de los emprendedores de micro y pequeñas empresas mexicanas del sector servicio de Mexicali, Baja California y Villahermosa, Tabasco, para identificar la importancia que para ellos tiene la innovación en el transcurso del tiempo. Lo anterior se logró a través de un estudio cuantitativo de alcance descriptivo, exploratorio y correlacional. Como parte de los resultados se tiene que a través de estas estrategias se coadyuva a potenciar un emprendimiento innovador, facilitando la generación de nuevas ideas y mejorando su capacidad de innovar para lograr productos o servicios escalables y de mayor valor, llevando la empresa a niveles competitivos e impulsando su desarrollo de forma sostenible. Se concluye que este esfuerzo debe ser permanente, pues existe una relación positiva y significativa entre la innovación y las acciones en el desarrollo de la misma, no sólo para la empresa sino para la entidad donde estas se ubican.

Palabras Clave: emprendimiento, innovación, micro y pequeñas empresas.

\section{Introducción}

La innovación y el emprendimiento son variables de la competitividad estratégica que están inmersas en las sociedades actuales, especialmente en ámbitos empresariales. Se consideran importantes instrumentos para avanzar en la mejora continua y están estrechamente relacionadas entre sí, representando factores clave para el rápido crecimiento de las empresas y de las naciones. Lo anteriormente expresado, se sustenta a partir del siguiente párrafo:

El emprendimiento se inicia con una idea que puede ser nueva o para mejorar un producto, proceso o servicio (De la Fuente, Vera \& Cardelle, 2012), la cual se gesta y concreta en un modelo y plan de negocio. La intención es llevar adelante ese negocio y ejecutarlo, lo que requiere ser proactivo (López, 2010), siendo fundamental adquirir conocimientos para emprender con éxito (Pico, Nuño \& Martínez, 2010; Urueña-López \& Hidalgo-Nuchera, 2013) en un ambiente donde proyectos empresariales se consideran clave a la innovación y el crecimiento económico (Minniti, Bygrave \& Autio, 2006; López-Torres, Maldonado, Pinzón \& García, 2015). 
Dado que la innovación es un proceso en red en el que participan todas las partes (Ríos \& Marroquín, 2012; Ahumada \& Perusquia, 2015), la cooperación entre los entornos científico y empresarial constituye una necesidad explícita que necesita ser mejorada (López-Torres et al., 2015), siendo esencial fomentar la innovación para impulsar la productividad y crear nuevas fuentes de crecimiento (Urueña-López \& Hidalgo-Nuchera, 2013). Todo esto se hace entendiendo que, a fin de poner en marcha proyectos innovadores, se requiere invertir en Investigación y Desarrollo (I\&D), actividad que involucra tanto a la iniciativa privada, al gobierno, a las universidades y a las instituciones de apoyo al emprendimiento (Etzkowitz, 2003; Rodeiro-Pazos \& Calvo-Babio, 2012).

En este tenor, el gasto interno bruto en I\&D en México representa un bajo porcentaje. De acuerdo a la OCDE (Organización para la Cooperación y el Desarrollo Económico), éste alcanzó apenas el 0.44\% del PIB (Producto Interno Bruto) en el 2009 -prácticamente la mitad de lo que invierte Turquía, con $0.85 \%$ del PIB, y muy por debajo de países como Corea del Sur, con el $3.6 \%$, Finlandia, con el 4\%, o Israel, con el 4.3\%- (Guzmán, López-Herrera \& Venegas-Martínez, 2012; Alcázar-Zamacona \& Haro-Zea, 2013). Entre los diversos factores que explican la debilidad de la innovación en México y limitan la inversión en I\&D están un marco legal deficiente y limitaciones de gobernabilidad en el sistema de innovación. En contraparte, se realizan esfuerzos para superar estas debilidades como la comercialización de la investigación pública y la creación de condiciones para el emprendimiento innovador (OCDE, 2012).

El estado mexicano se está transformando y la matriz de soporte a la innovación se está reconfigurando, tomando en cuenta lo expuesto en el estudio realizado por Estrada y Pacheco-Vega (2009), el cual afirma que el sistema de innovación mexicano y su marco institucional están en proceso de maduración evolutiva y de franco aprendizaje. En este sentido, emprendedores de micro y pequeñas empresas deben estar conscientes de la importancia que representan estas organizaciones en la generación de empleo y en la contribución al PIB en la economía nacional (Restrepo \& Vanegas, 2015). De igual manera, se consolidan como una posibilidad de mejoramiento de la competitividad de un país (Mora-Riapira, Vera-Colina \& Melgarejo-Molina, 2015).

Por su parte, De Fuentes (2007) enfatiza la importancia que reviste el conocimiento y la información sobre un emprendimiento innovador. Por ello, factores como: la formación y experiencia del emprendedor, la incorporación de tecnología, las colaboraciones que establezcan con otras empresas, las capacidades organizacionales y las actividades de innovación y aprendizaje son características que, combinadas, generan diferentes patrones que les permiten a las micro y pequeñas empresas ser competitivas. Por tal motivo, 
resulta esencial fomentar emprendimientos de calidad y facilitar las ideas innovadoras y su puesta en marcha en proyectos emprendedores (Dosal, Gutiérrez \& Saracho, 2010; Naranjo-Valencia \& Calderón-Hernández, 2015).

Bajo este contexto, la investigación tuvo como objetivo contrastar las acciones enfocadas a la innovación, a través de un estudio cuantitativo realizado a emprendedores de micro y pequeñas empresas mexicanas del sector servicio para identificar la importancia que para ellos tiene la innovación. Como parte del trabajo documental, se revisó literatura a fin de sustentar las siguientes líneas teóricas: 1) Innovación, eje central en el emprendimiento, y 2) La innovación como ventaja estratégica para emprendedores de micro y pequeñas empresas.

\section{Innovación, eje central en el emprendimiento}

Dyer, Gregersen y Christensen (2009) analizaron la relación que existe entre emprendimiento e innovación, donde establecen que los emprendedores innovadores cuentan con una inteligencia creativa que les permite ser agentes de cambio en la generación de proyectos de modelo de negocio exitosos.

Como parte de sus potencialidades, el emprendimiento se aboca a la creación de empresas y contribuye a elevar el nivel socioeconómico de las naciones porque permite la generación de ingresos, la disminución de la pobreza y el bienestar ciudadano (Santillán, Gaona \& Hernández, 2015). Por consiguiente, para iniciar una iniciativa empresarial es clave tener en cuenta las oportunidades de innovación y experimentar ideas propias con flexibilidad y mente abierta; siendo necesario repensar el crecimiento económico fundamentado en la innovación de negocios (Pico et al., 2010).

En este contexto, ha cobrado auge el emprendimiento innovador como aliado de las economías nacionales, tanto en países europeos como en América Latina y Estados Unidos (Estrategia \& Negocios, 2013) puesto que, en un mundo globalizado, el nivel de competitividad es elevado y se requiere crear valor en las organizaciones (López-Torres et al., 2015; Santillán et al., 2015), siendo la innovación uno de los principales aspectos para lograrlo.

El Manual de Oslo (OCDE, 2005) define la innovación como la introducción de un nuevo o significativamente mejorado producto -ya sea bien o servicio-, un proceso, o un método de comercialización u organizativo; es decir, acciones de tipo tecnológico, operacional, administrativo o estratégico, que promueven la mejora de productos o servicios (Ahumada \& Perusquia, 2015). En correspondencia, un empresario innovador es un individuo con ideas de cambio y de mejora continua (Pomar, Rendon \& Ramírez, 2013).

Esta relación entre emprendimiento y el proceso de innovación la fundamenta la OCDE (2005), haciendo una clasificación de los emprendedores de acuerdo a Schumpeter, Kirzner, Knight y Drucker en la cual se describe al emprendedor como: 1) disruptor, considerándolo el principal 
actor dentro del proceso de innovación, iniciador del cambio y generador de nuevas oportunidades; 2) cazador de oportunidades, cuando el emprendedor identifica una oportunidad de negocio que para otros pasa desapercibida; 3 ) tomador de riesgos, cuando el emprendedor es innovador simplemente por atreverse a experimentar en el mercado y aceptar el alto riesgo del fracaso; y 4) emprendedor con capacidad para reasignar recursos cuando destina lo existente en nuevas oportunidades de generación de riqueza (Dosal et al., 2010).

Asimismo, propiciar un ambiente oportuno es clave para que la innovación se desarrolle con éxito y se aprovechen los recursos de manera eficiente (Mathison, Gándara, Primera \& García, 2007; De la Fuente et al., 2012; Gantman, 2013; Urueña-López \& Hidalgo-Nuchera, 2013); en este caso, se requieren emprendedores abiertos al aprendizaje y con pensamiento sistémico, proactivos, innovadores e inspiradores, que potencien las colaboraciones, promuevan la innovación, fomenten la confianza, la sana confrontación, y asuman riesgos controlados (Naranjo-Valencia \& CalderónHernández, 2015).

En consecuencia, Dyer, Gregersen y Christensen (2009) establecieron que los emprendedores innovadores requieren cultivar habilidades de descubrimiento a través de: asociar, preguntar, observar, experimentar, y la creación de redes; las cuales, en su conjunto, conforman lo que llamaron el $A D N$ del innovador. No importa que no se nazca con ellas, dado que se pueden desarrollar. En este sentido, la asociación ayuda a los innovadores a cultivar nuevos puntos de vista; el interrogatorio permite a los innovadores salir de la situación actual y considerar nuevas posibilidades; a través de la observación, los innovadores detectan pequeños detalles de comportamiento en las actividades de los clientes, proveedores y otras empresas que sugieren nuevas formas de hacer las cosas; en la experimentación, se prueban nuevas experiencias y se explorara el mundo; y a través de redes con personas de diversos orígenes ganan radicalmente diferentes perspectivas.

\section{La innovación como ventaja estratégica para emprendedores de micro y pequeñas empresas}

La capacidad de los emprendedores para innovar y mejorar en sus empresas resultan aspectos importantes que posibilitan el logro de una ventaja competitiva; una vez obtenida, se requiere de una mejora incesante para mantenerse en competencia (Díaz, 2009). Por ello, es fundamental que creen valor a través de la realización de actividades de manera diferente a sus rivales (Porter, 1990).

En este sentido, la innovación se convierte en uno de los factores clave de consolidación en el entorno global competitivo (Ahumada \& Perusquia, 2015); pero adquirir estas ventajas y sacarle el máximo provecho en su 
operatividad implica también gestionar el proceso de aprendizaje organizacional (Mendoza \& Valenzuela, 2013) con el fin de impulsar la expansión, la creación de conocimientos y el uso de tecnologías innovadoras en empresas emprendedoras a nivel mundial, y que logren ubicarse en un mercado cada vez más competitivo (Ríos \& Marroquín, 2012).

En este contexto, el enfoque basado en la innovación y el uso de ventajas estratégicas permite a las organizaciones ser eficientes y mejorar su rendimiento en diferentes aspectos, tales como: la productividad, la rentabilidad y la calidad (Caro, Leyva \& Manzanilla, 2011; Romo \& Rivas, 2012). Del mismo modo, los emprendedores de las micros y pequeñas empresas deben desarrollar conceptos de negocio a partir de modelos de emprendimiento innovadores, considerando a la planeación estratégica como otro componente organizacional (Castaño, 2011).

\section{Metodología}

La presente investigación se basó en una metodología con enfoque cuantitativo de alcance descriptivo, exploratorio y correlacional, con el objeto de contrastar las acciones enfocadas a la innovación a través de un estudio cuantitativo realizado a emprendedores de micro y pequeñas empresas mexicanas del sector servicio, nacidas en las ciudades de Mexicali, Baja California y Villahermosa, Tabasco para identificar la importancia que para ellos tiene la innovación. De dicho objetivo, se desprenden las variables: innovación y acciones de innovación.

Para la recolección de datos, se recurrió a un instrumento validado en sus dos dimensiones: confiabilidad y validez. Dicho instrumento fue un cuestionario que formó parte de un estudio de innovación y emprendimiento en Chile (Kantis, 2008), el cual ya estaba validado y fue adecuado en base a las variables descritas en esta investigación. Su proceso de validación consistió en el análisis factorial de correspondencias múltiples, a través del cual se conformaron ejes factoriales cuya determinación permite concentrar la mirada en aquellas variables y modalidades que más aportan a explicar la semejanza y variabilidad en el comportamiento de las empresas examinadas.

\section{Planteamiento de hipótesis}

De acuerdo a la investigación documental y en relación al objetivo y variables de estudio, se plantean las siguientes hipótesis:

Hipótesis 1. Existe una relación positiva y significativa entre la innovación y las acciones en el desarrollo de la misma.

En este contexto, la literatura manifiesta que un empresario innovador procura el cambio y la mejora continua (Pomar et al., 2013), es abierto al aprendizaje, con pensamiento sistémico y proactivo (Dosal et al., 2010; Naranjo-Valencia \& Calderón-Hernández, 2015) manifestando una fuerte 
relación del emprendimiento con el desarrollo de la innovación (Dyer et al., 2009).

Hipótesis 2. Una región con empresas innovadoras, desarrolla mayores acciones en la innovación.

Dado que la innovación es un factor estratégico para posicionarse en un mercado global y dinámico (Ahumada \& Perusquia, 2015), impulsar empresas innovadoras a nivel mundial permite fortalecer un mercado cada vez más competitivo (Castaño, 2011; Ríos \& Marroquín, 2012).

\section{Población y tamaño de muestra}

Con un nivel de respuesta limitado, la población sujeta a estudio se calculó en base a un muestreo estratificado, teniendo una población de cerca de 2,000 empresas y discriminando aquellas en las que no fue posible contar con la información. La muestra fue representativa, teniendo los siguientes elementos: nivel de confianza del $85 \%$ y $19 \%$ de margen de error, resultando una muestra a encuestar de un $60 \%$ de empresas en Villahermosa y $40 \%$ en Mexicali y considerando un perfil de innovación basado en los criterios del Manual de Oslo (2005), los cuales son: 1) generar conocimientos nuevos, 2) transferir o adaptar tecnología, y 3) introducir nuevo proceso, producto o servicio (Tabla 1).

Tabla 1. Perfil de la muestra

\begin{tabular}{|c|c|c|c|c|c|c|}
\hline \multirow{3}{*}{ ESTRATO } & \multirow{3}{*}{ GIRO DE LA EMPRESA } & \multirow{2}{*}{\multicolumn{2}{|c|}{ CIUDAD }} & \multicolumn{3}{|c|}{ La empresa aporta a la innovación: } \\
\hline & & & & \multirow{2}{*}{$\begin{array}{c}\text { NUEVOS } \\
\text { CONOCI } \\
- \\
\text { MIENTO } \\
\text { S }\end{array}$} & \multirow{2}{*}{$\begin{array}{l}\text { TRANSFE- } \\
\text { RENCIA DE } \\
\text { TECNOLO } \\
\text { GÍA }\end{array}$} & \multirow{2}{*}{$\begin{array}{c}\text { NUEVO } \\
\text { PROCESO } \\
\text { PRODUCT } \\
\text { O O } \\
\text { SERVICIO }\end{array}$} \\
\hline & & VH & $\begin{array}{c}\text { MX } \\
\text { L }\end{array}$ & & & \\
\hline \multirow[t]{2}{*}{ Estrato 1} & $\begin{array}{l}\text { Laboratorio de diagnóstico clínico e } \\
\text { imagenología, incluyendo medicina } \\
\text { laboral y estudios de genética. }\end{array}$ & $\mathrm{X}$ & & $\mathrm{X}$ & $\mathrm{X}$ & $\mathrm{X}$ \\
\hline & $\begin{array}{c}\text { Clínica de salud nutricional y } \\
\text { deportiva. }\end{array}$ & & $\mathrm{X}$ & & & $X$ \\
\hline Estrato 2 & $\begin{array}{c}\text { Servicio de construcción de } \\
\text { ingeniería civil y obra pesada con } \\
\text { tecnología de punta y reportes en } \\
\text { tiempo real. }\end{array}$ & $\mathrm{X}$ & & & $\mathrm{X}$ & $\mathrm{X}$ \\
\hline \multirow{3}{*}{ Estrato 3} & $\begin{array}{c}\text { Servicio de decoración empleando } \\
\text { arreglos frutales con diseños } \\
\text { personalizados. }\end{array}$ & $\mathrm{X}$ & & & & $X$ \\
\hline & $\begin{array}{l}\text { Asesoría en decoración para } \\
\text { eventos utilizando material } \\
\text { reciclado y productos naturales. }\end{array}$ & $\mathrm{X}$ & & $\mathrm{X}$ & & $\mathrm{X}$ \\
\hline & Servicio integral de fotografías. & & $\mathrm{X}$ & & $\mathrm{X}$ & $\mathrm{X}$ \\
\hline Estrato 4 & $\begin{array}{l}\text { Consultoría, análisis y desarrollo de } \\
\text { software. }\end{array}$ & & $\mathrm{X}$ & $\mathrm{X}$ & & $\mathrm{X}$ \\
\hline
\end{tabular}




\begin{tabular}{|c|c|c|c|c|c|c|}
\hline & $\begin{array}{c}\text { Desarrollo de software } \\
\text { personalizado }\end{array}$ & & $X$ & & $\mathrm{X}$ & $\mathrm{X}$ \\
\hline & $\begin{array}{l}\text { Desarrollo de software para } \\
\text { investigación de mercados. }\end{array}$ & & $\mathrm{X}$ & $\mathrm{X}$ & & $\mathrm{X}$ \\
\hline & $\begin{array}{c}\text { Diseño de Software para } \\
\text { aplicaciones en dispositivos } \\
\text { móviles y videojuegos. }\end{array}$ & & $\mathrm{X}$ & $\mathrm{X}$ & & $\mathrm{X}$ \\
\hline \multirow{3}{*}{ Estrato 5} & $\begin{array}{c}\text { Consultoría, asesoría y capacitación } \\
\text { empresarial. }\end{array}$ & $X$ & & $X$ & & $\mathrm{X}$ \\
\hline & $\begin{array}{l}\text { Consultoría y diseño de imagen } \\
\text { corporativa e impresión en 3D. }\end{array}$ & $\mathrm{X}$ & & $X$ & $\mathrm{X}$ & $\mathrm{X}$ \\
\hline & Diseño Gráfico. & $\mathrm{X}$ & & & & $\mathrm{X}$ \\
\hline \multirow{2}{*}{ Estrato 6} & $\begin{array}{l}\text { Servicio de lavandería a domicilio } \\
\text { mediante una aplicación WEB. }\end{array}$ & $\mathrm{X}$ & & & & $\mathrm{X}$ \\
\hline & $\begin{array}{l}\text { Servicio de limpieza de prendas con } \\
\text { productos biodegradables. }\end{array}$ & $\mathrm{X}$ & & & $\mathrm{X}$ & $\mathrm{X}$ \\
\hline
\end{tabular}

Fuente: Elaboración propia, 2018.

La información anterior permitió comparar el comportamiento de emprendedores en dos ciudades que pertenecen a entidades fronterizas situadas en distintas regiones, con diferencias en cuanto a su extensión, desarrollo, potencial económico y competitividad; esta última puede resultar ser un indicador de las acciones realizadas en el ámbito de la innovación a nivel global. Al respecto, el Ranking de la Competitividad de los Estados Mexicanos coloca a Baja California como la novena entidad más competitiva del país, mientras que Tabasco ocupa el lugar 18. En cuanto al índice de Eficiencia de Negocios, Baja California se encuentra en la posición nueve y Tabasco en la 12, y el índice de potencial de innovación ubica a Baja California en el número 14 de México (Campos, 2012). Esta entidad federativa se encuentra entre los estados más desarrollados e industrializados de México (Ramírez, Bernal \& Fuentes, 2013). Dicho panorama no es así de alentador para Tabasco porque, a pesar de que cuenta con importantes riquezas naturales y una posición geográfica estratégica en el sureste mexicano, tiene un bajo crecimiento económico y elevada tasa de desempleo. Ante ello, una actitud emprendedora enfocada a la creación de empresas innovadoras contribuiría a la reactivación económica y progreso de la entidad (Rodríguez, Hernández \& Martínez, 2017).

Análisis de hipótesis

El análisis de las hipótesis se realizó mediante la corrida del software estadístico SPSS y se calcularon las correlaciones para cada caso a través del coeficiente $r$ de Pearson.

Para la hipótesis 1 que plantea: "existe una relación positiva y significativa entre la innovación y las acciones en el desarrollo de la misma", 
se obtuvo una correlación significativa de 0.705 al 1\% (Tabla 4). Para el caso de la hipótesis 2: "una región con empresas innovadoras desarrolla mayores acciones en la innovación", se calcularon las medias entre las motivaciones por iniciar el emprendimiento y el desarrollo de la innovación, obteniendo que no es significativa la diferencia entre dichos promedios en las ciudades muestreadas (Tablas 5a y 5b).

En consecuencia, desarrollar innovación es importante para todos los emprendedores del estudio, y forma parte de las razones que los motivaron a iniciar sus emprendimientos; adicional a esto, sin importar cuál sea la región, la innovación permanece como elemento estratégico en los emprendimientos.

\section{Resultados}

Después del análisis de los datos recolectados en el trabajo de campo, detallado ampliamente en la metodología de la investigación, se obtienen resultados enfocados a determinar las acciones llevadas a cabo por los emprendedores para desarrollar innovación.

Como resultado del análisis (Gráfica 1) y derivado de las razones que originó que los emprendedores iniciaran sus emprendimientos, se obtuvo que la detección de una necesidad en el mercado a través de la generación de un producto o servicio (53.3\%), fue el principal motivo.

Gráfica 1. Razones por las que inician los emprendimientos

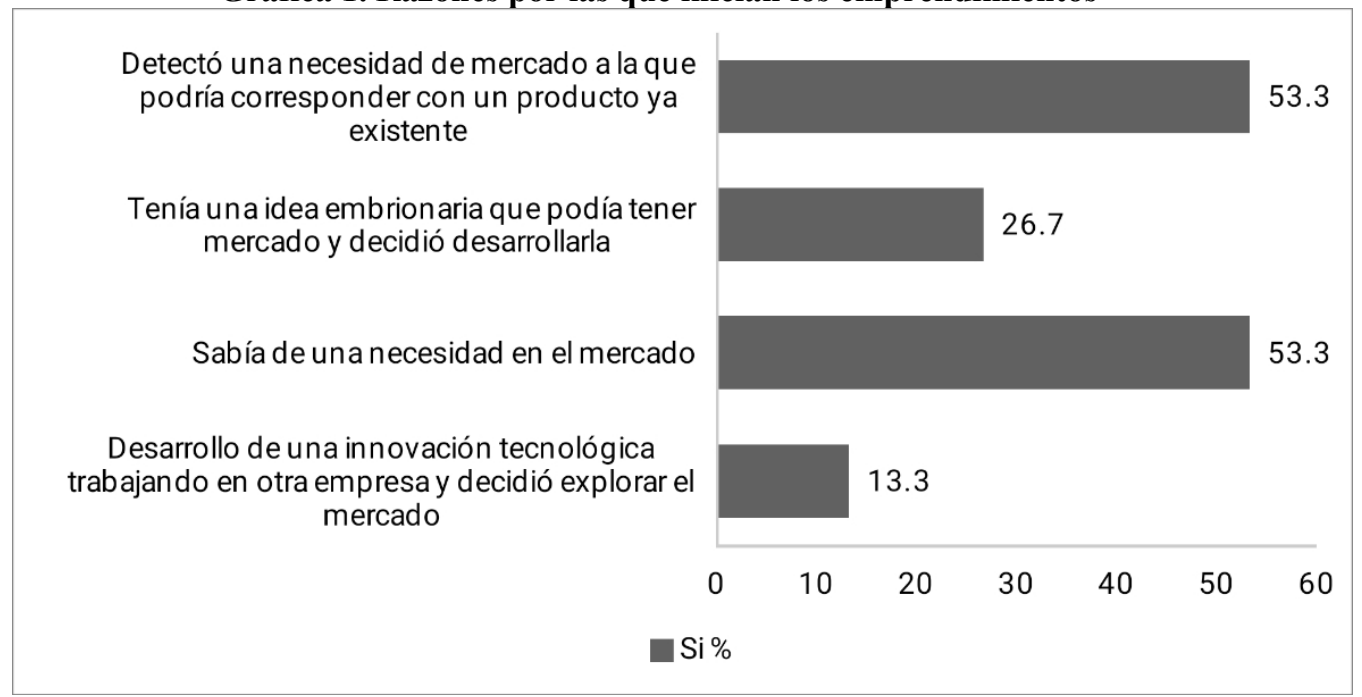

Fuente: Elaboración propia, 2018.

En este contexto, hubo una variación de las motivaciones para iniciar su proyecto de negocio en sus comienzos con respecto al momento en que se recolectaron los datos del estudio (Gráfica 2). De lo más significativo, se tiene que al inicio el $100 \%$ de los emprendedores tenían como motivación las oportunidades para promover un producto/servicio innovador o satisfacer una 
necesidad o problema que no era atendido por alguna empresa en el extranjero, y al paso del tiempo se convirtió en tan solo el $26.67 \%$, lo que implica una disminución del $73.33 \%$. También, se contrastó que hubo un incremento del $20 \%$ en los intereses de los emprendedores por competir con un producto diferenciado, con factores como prestaciones, calidad y servicio. Adicionalmente, se tiene que la única variable que no tuvo cambio (26.67\%) fue el hecho de proveer bienes y/o servicios a empresas que los producían internamente.

Gráfica 2. Motivación en la que se basaron los emprendedores su proyecto de negocio al nacer y actualmente

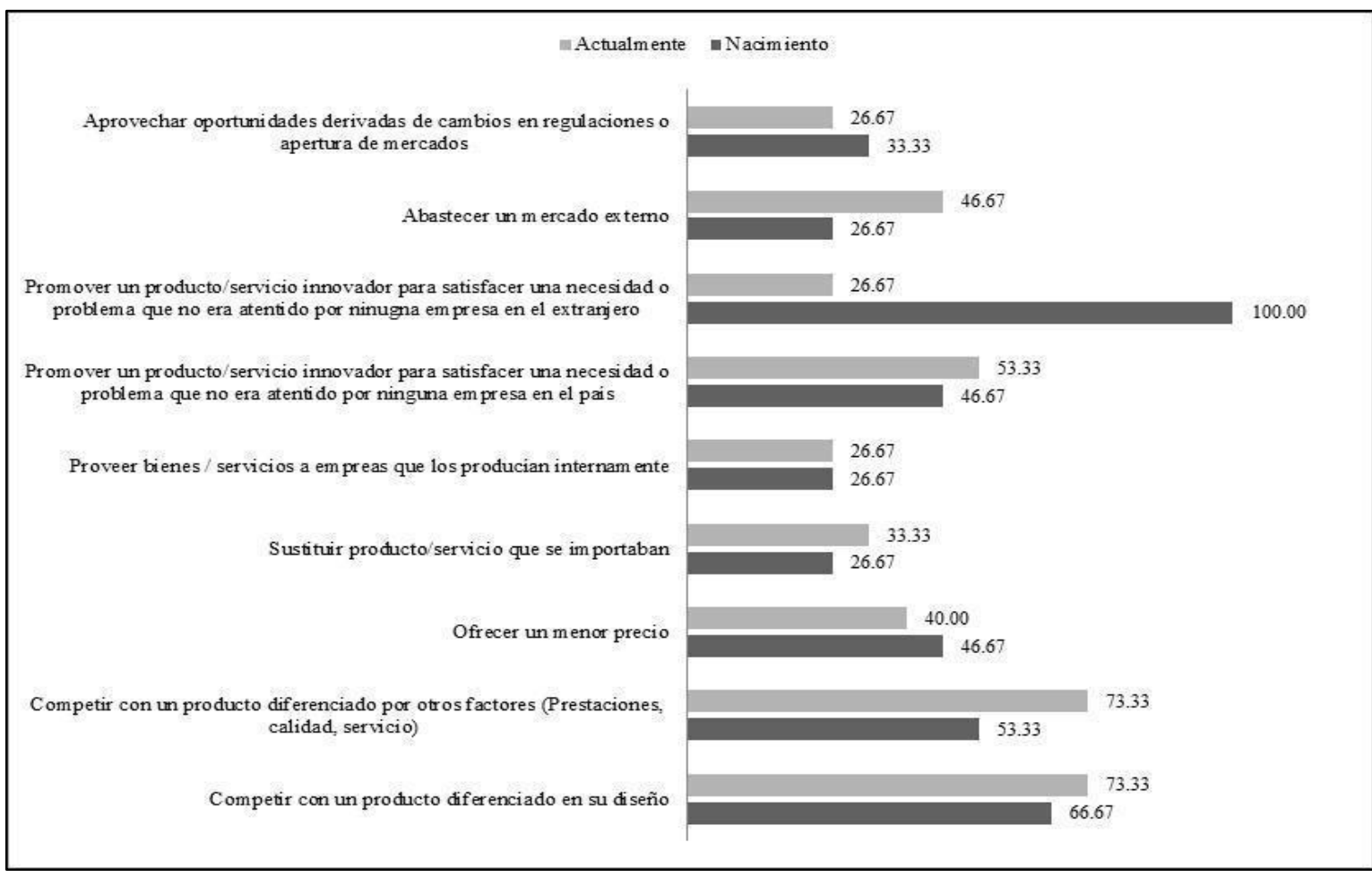

Fuente: Elaboración propia, 2018.

En cuanto a los resultados relacionados con la inversión para implementar y desarrollar innovación, las áreas donde los emprendedores llevaron a cabo inversiones se desglosaron y calificaron bajo la siguiente escala de Likert: 1- no se obtuvieron resultados, 2- resultados parciales, 3resultados altos, y 4- los resultados se verán a futuro. Del anterior análisis sobre las áreas en las cuáles los emprendedores focalizan sus innovaciones (Tabla 2), así como el alcance de su inversión en cuestión de obtención de resultados, se obtuvo que desarrollar la imagen comercial/marca y adquirir nuevos equipos para ganar calidad/productividad fueron las que mejor promedio obtuvieron al ser de 2.92 y 2.91 respectivamente; desarrollar 
mercados externos y adquirir nuevas instalaciones, resultaron los más bajos promedios con 2.33 y 2.40 en cada caso.

Tabla 2. Grado de consecución de los resultados en las áreas invertidas para el desarrollo de innovación

\begin{tabular}{|c|c|c|c|}
\hline ÁREAS DE INVERSIÓN & SUMA & PROMEDIO & $\begin{array}{c}\text { DESVIACIÓN } \\
\text { ESTANDAR }\end{array}$ \\
\hline Desarrollar mercado externos & 5 & 2.33 & 1.24 \\
\hline Desarrollar y lanzar nuevos productos & 38 & 2.71 & .958 \\
\hline Desarrollar la imagen comercial/ marca & 38 & 2.92 & .615 \\
\hline Desarrollar canales de comercialización & 30 & 2.5 & .957 \\
\hline $\begin{array}{c}\text { Desarrollar proyectos de I\&D } \\
\text { Adquirir nuevos equipos para ganar calidad/ } \\
\text { productividad }\end{array}$ & 30 & 2.5 & 1.04 \\
\hline $\begin{array}{c}\text { Adquirir nuevos equipos para ampliar la capacidad de } \\
\text { producción }\end{array}$ & 36 & 2.91 & .514 \\
\hline $\begin{array}{c}\text { Introducir tecnologías de la información y } \\
\text { comunicaciones }\end{array}$ & 34 & 2.77 & .576 \\
\hline Adquirir nuevas instalaciones & 24 & 2.83 & .799 \\
\hline Adquirir instalaciones edificadas disponibles & 19 & 2.71 & .917 \\
\hline
\end{tabular}

Fuente: Elaboración propia, 2018.

En la búsqueda para generar sostenibilidad a través de esfuerzos en innovación, los emprendedores están conscientes de que deben destinar un porcentaje de su reinversión al área de Investigación y Desarrollo (I \& D). Esto se sustenta considerando que $80 \%$ destinan en promedio el $21 \%$ de los ingresos por ventas a inversión en actividades de I \& D. De la mano de la inversión destinada al área de I \& D, se detectó que el 93.3\% tienen como parte de su filosofía de trabajo el destinar un porcentaje de las horas laborales a actividades de diseño en I \& D.

El resultado de la inversión en tiempo y dinero en diseño de I \& D ha sido que el $93.3 \%$ de los emprendedores lograran la producción de ideas nuevas -concretadas- y que las mismas han derivado en innovaciones de producto, servicio o proceso.

En el rubro de diseño, los emprendedores respondieron sobre el grado de importancia que tienen para ellos los esfuerzos destinados a diferentes aristas que conforman esta variable, mediante una escala de Likert donde, 1 es No importante, 2 Poco importante, 3 Importante y 4 Muy importante. Para poder establecer cuáles fueron los rubros de mayor trascendencia para los emprendedores se utilizaron promedios, y los más cercanos a 4 son aquellos que se consideran de mayor peso (Tabla 3); siendo la generación de ideas para investigación y desarrollo, con un promedio de 3.8, la más alta y la de menor importancia fue la que corresponde al apartado del embalaje. 
Tabla 3. Grado de importancia de las siguientes actividades en relación a las acciones en área de diseño

\begin{tabular}{|c|c|}
\hline ÁREAS DE DISEÑO & PROMEDIO \\
\hline Generación de ideas, I\&D & 3.8 \\
\hline Producción de prototipos & 3.14 \\
\hline Desarrollo del producto & 3.87 \\
\hline Proceso de producción & 3.69 \\
\hline Embalaje & 3.1 \\
\hline Mercadotecnia & 3.73 \\
\hline A nivel global/general de la empresa & 3.73 \\
\hline
\end{tabular}

Fuente: Elaboración propia, 2018.

En cuanto a los resultados de las pruebas de hipótesis y correlaciones, la Hipótesis 1 plantea que: existe una relación positiva y significativa entre la innovación y las acciones en el desarrollo de la misma, para la cual se obtuvo una correlación significativa a través del coeficiente $r$ de Pearson, teniendo como resultado 0.705 y significativa al $1 \%$ (Tabla 4 ).

Tabla 4. Correlaciones

\begin{tabular}{|c|c|c|r|}
\multicolumn{2}{c|}{} & \multicolumn{1}{c|}{$\begin{array}{c}\text { MOTIVACIÓN AL INICIAR } \\
\text { EL EMPRENDIMIENTO }\end{array}$} & $\begin{array}{c}\text { DESARROLLO DE } \\
\text { INNOVACIÓN }\end{array}$ \\
\hline $\begin{array}{c}\text { MOTIVACIÓN AL } \\
\text { INICIAR EL } \\
\text { EMPRENDIMIENTO }\end{array}$ & $\begin{array}{c}\text { Correlación de } \\
\text { Pearson }\end{array}$ & 1 & $.705^{* *}$ \\
\cline { 2 - 4 } $\begin{array}{c}\text { DESARROLLO DE } \\
\text { INNOVACIÓN }\end{array}$ & $\begin{array}{c}\text { Sig. (bilateral) } \\
\text { Correlación de } \\
\text { Pearson }\end{array}$ & $.705^{* *}$ & .003 \\
\cline { 2 - 4 } & Sig. (bilateral) & .003 & 1 \\
\hline \multicolumn{2}{|c|}{$* *$ La correlación es significativa en el nivel 0,01 (bilateral). } \\
\hline
\end{tabular}

Fuente: Elaboración propia, 2018.

En este contexto, sin importar cuáles fueron las causas que motivaron a los emprendedores en sus inicios, el desarrollo de la innovación es importante para ellos y está implícita en los motivos que dieron origen a los emprendimientos.

En cuanto a la Hipótesis 2 que plantea que: una región con empresas innovadoras, desarrolla mayores acciones en la innovación, la prueba de hipótesis (Tablas 5a y 5b) determinó que sin importar la región, no es significativa la diferencia entre los promedios de la motivación por iniciar el emprendimiento, ni tampoco es significativa la diferencia entre los promedios del desarrollo de la innovación. 
Tabla 5a.

Prueba de Hipótesis: Motivación al Iniciar el Emprendimiento

\begin{tabular}{|c|c|c|}
\hline Mexicali & Villa Hermosa & \\
\hline 2.64 & $\begin{array}{r}3.01 \\
\end{array}$ & mean \\
\hline 1.17 & 1.2 & std. dev. \\
\hline 6 & 9 & n \\
\hline & 13 & df \\
\hline & -0.37000 & difference (Mexicali - Villa Hermosa) \\
\hline & 1.41265 & pooled variance \\
\hline & 1.18855 & pooled std. dev. \\
\hline & 0.62642 & standard error of difference \\
\hline & 0 & hypothesized difference \\
\hline & -0.59 & $\mathrm{t}$ \\
\hline & .5649 & p-value (two-tailed) \\
\hline
\end{tabular}

Fuente: Elaboración propia, 2018.

Tabla 5b.

Prueba de Hipótesis: Desarrollo de la Innovación

\begin{tabular}{|c|c|c|}
\hline Mexicali & Villa Hermosa & \\
\hline 3.5 & 3.67 & mean \\
\hline 0.77 & 0.7 & std. dev. \\
\hline 6 & 9 & $\mathrm{n}$ \\
\hline & 13 & df \\
\hline & -0.17000 & difference (Mexicali - Villa Hermosa) \\
\hline & 0.52958 & pooled variance \\
\hline & 0.72772 & pooled std. dev. \\
\hline & 0.38354 & standard error of difference \\
\hline & 0 & hypothesized difference \\
\hline & -0.44 & $\mathrm{t}$ \\
\hline & .6649 & p-value (two-tailed) \\
\hline
\end{tabular}

Fuente: Elaboración propia, 2018.

Tomando en cuenta lo antes expuesto, al contrastar dichas variables en las ciudades estudiadas se obtuvo una media de 2.64 en Mexicali y 3.01 en Villahermosa en cuanto al grado de innovación que motivó a los emprendedores; contrastando con el desarrollo de la innovación con una media de 3.5 en Mexicali y 3.67 en Villahermosa. Estos promedios resultaron cercanos al 4, valor de mayor importancia que le dio el emprendedor a cada variable al momento de la recolección de datos. 


\section{Conclusión}

El trabajo de investigación desarrollado permitió sustentar que la innovación como parte de los emprendimientos resulta un factor diferenciador importante. En este sentido, el 100\% de los emprendedores encuestados coincidieron que innovar es un elemento esencial para competir en mercados dinámicos, por lo que su compromiso en el proceso de la innovación es constante.

Adicional a esto, las razones por las cuales iniciaron sus emprendimientos se relacionan con su motivación por desarrollar proyectos con base en la innovación, ya que los emprendedores objetos del estudio están convencidos de que ello les permitirá crecer y consolidarse. Dichos hallazgos se obtuvieron tanto para Villahermosa como para Mexicali, donde, aun siendo ciudades con características muy diferentes, en ambas se reconoce el valor de la innovación por parte de los emprendedores.

En relación a las hipótesis planteadas es posible afirmar que los emprendedores en ambas entidades federativas se encuentran comprometidos con realizar acciones en innovación para ser sostenibles en el tiempo; por tanto, aun considerando que las vocaciones y condiciones de cada entidad son diferentes, en ambas existe el interés por parte de los emprendedores en desarrollar micro y pequeñas empresas centradas en la innovación, al no existir una gran discrepancia en los promedios generados.

De igual manera, es importante destacar que las acciones enfocadas a la innovación, representan esfuerzos permanentes principalmente para los emprendedores, cuyos proyectos se concretan en la creación de micro y pequeñas empresas que, dadas sus características, requieren innovar para permanecer en mercados dinámicos, y que además les ofrece una ventaja competitiva.

Para finalizar, a partir de la investigación realizada se detectó que existen grandes áreas de oportunidad para fortalecer esta línea con investigación aplicada, por lo que para futuras investigaciones, se considera estudiar el impacto del seguimiento a proyectos emprendedores, liderados por emprendedores innovadores.

\section{References}

1. Ahumada, E. \& Perusquia, J. (2015). Inteligencia en negocios: estrategia para el desarrollo de competitividad en empresas de base tecnológica. Contaduría y Administración, 61(2016), 127-158.

2. Alcázar-Zamacona, J.P. \& Haro-Zea, K.L. (2013). Análisis cuantitativo de patentes en México. Global Conference On Business \& Finance Proceedings, 8(1), 830-837. 
3. Campos, N. (2012). Ranking de la Competitividad de los Estados Mexicanos. Instituto Mexicano para la Competitividad. Recuperado de http://imco.org.mx.

4. Caro Encalada, M.J., Leyva Morales, C.E. \& Manzanilla, R.V. (2011). Calidad de las tecnologías de la información y competitividad en los hoteles de la península de Yucatán. Contaduría y Administración, 235 (septiembre-diciembre 2011), 121-146.

5. Castaño Ramírez, A. (2011). Planeación estratégica en la Pyme de Bogotá, Orientación al futuro y evasión de la incertidumbre. Contaduría y Administración, 235 (septiembre-diciembre 2011), 147173.

6. De Fuentes, C. (2007). Derramas de conocimiento y capacidades de absorción: el caso de las pymes de maquinados industriales de Querétaro. Tesis doctoral, México, DF, Universidad Autónoma Metropolitana - Xochimilco.

7. De la Fuente, J., Vera, M. \& Cardelle, M. (2012). Aportaciones de la Psicología de la Innovación y del Emprendimiento a la Educación, en la Sociedad del Conocimiento. Electronic Journal of Research in Educational Psychology, 10(28), 941-966.

8. Díaz Fernández, I. (2009). Enfoque de Porter y de la teoría basada en los recursos en la identificación de la Ventaja Competitiva: ¿contraposición o conciliación? Economía y Desarrollo, 144(1), 101114.

9. Dosal, C., Gutiérrez, C. \& Saracho, A. (2010). ¿Quiénes son los emprendedores innovadores mexicanos?, OCDE. Recuperado de http://observatorioredesempresariales.files.wordpress.com/2011/06/e mprendedores.pdf.

10. Dyer H. J., Gregersen B. H. \& Christensen M. C. (2009). The Innovator's DNA. Harvard Bussines Review.

11. Estrada, S. \& Pacheco-Vega, R. (2009). Sistemas y políticas de investigación, desarrollo e innovación. Algunas propuestas. Espiral, 15(44), 31-76.

12. Estrategia y Negocios. (2013). Emprendedores Innovadores Mexicanos, El perfil de los emprendedores en México. Academia Latinoamericana de Emprendedores. Recuperado de http://www.estrategiaynegocios.net/.

13. Etzkowitz, H. (2003). Innovation in innovation: The triple helix of university-industry-government relations. Social science information, 42(3), 293-337.

14. Gantman, E.R. (2013). Escuelas de negocios latinoamericanas, Un análisis empírico. Contaduría y Administración, 58 (3), julioseptiembre 2013, 279-298. 
15. Guzmán Chávez, Alenka G., López-Herrera, F. \& Venegas-Martínez, F. (2012). Un análisis de cointegración entre patentes y crecimiento económico en México, 1980-2008. Investigación económica, 71(281), 83-115.

16. Kantis, H. \& Díaz, S. (2008). Innovación y emprendimiento en Chile: una radiografía de los emprendedores dinámicos y de sus prácticas empresariales. Santiago, Chile.

17. López, A. (2010). La proactividad empresarial como elemento de competitividad. Ra Ximhai, 6(2), 303-312.

18. López-Torres, G.C., Maldonado, G., Pinzón C.S.Y. \& García, R. (2015). Colaboración y actividades de innovación en Pymes. Contaduría y Administración, 61(2016), 568-581.

19. Mathison, L., Gándara, J., Primera, C. \& García, L. (2007). Innovación: factor clave para lograr ventajas Competitivas. Ciencias Gerenciales, 3(7), 46-83. ISSN: 1856-1810.

20. Mendoza, J. \& Valenzuela, A. (2013). Aprendizaje, innovación y gestión tecnológica en la pequeña empresa: Un estudio de las industrias metalmecánica y de tecnologías de información en Sonora. Contaduría y Administración, 59(4), 253-284.

21. Minniti M., Bygrave W., \& Autio E. (2006). Global Report on HighGrowth Entrepreneurship. London: Babson College, London Business School and Global Entrepreneurship Monitor.

22. Mora-Riapira, E.H., Vera-Colina, M.A., \& Melgarejo-Molina, Z.A. (2015). Planificación estratégica y niveles de competitividad de las Mipymes del sector comercio en Bogotá. Estudios Gerenciales, 31(134), 79-87.

23. Naranjo-Valencia, J. C., \& Calderón-Hernández, G. (2015). Construyendo una cultura de innovación. Una propuesta de transformación cultural. Estudios Gerenciales, 31 (Abril-Junio 2015), 137-240.

24. OCDE. (2005). Manual de Oslo, Guía para la recogida e interpretación de datos sobre innovación.

25. OCDE. (2012). México, mejores políticas para un desarrollo incluyente. OCDE, septiembre 2012.

26. Pico B., Nuño, P. \& Martínez J.L. (2010). Modelo para Formación de Emprendedores basado en la Calidad Humana. Universidad Popular Autónoma de Puebla. México.

27. Pomar, S., Rendon, A. y Ramírez, H. (2013). Problemática en la gestión tecnológica en la micro y pequeña empresa. Dos estudios de caso. Revista Pistas Educativas.

28. Porter, M. (1990). La Ventaja Competitiva de las Naciones. Harvard Business Review. 
29. Ramírez Urquidy, M., Manuel Bernal, M. \& Fuentes, R. (2013). Emprendimiento y desarrollo manufactureros en las entidades federativas de México. Problemas del Desarrollo, 174 (44), julioseptiembre 2013, 167-195.

30. Restrepo, J. \& Vanegas, J. (2015). Internacionalización de las Pymes: análisis de recursos y capacidades internas mediante lógica difusa. Contaduría y Administración, 60, 836-863.

31. Ríos, H. \& Marroquín, J. (2012). Innovación tecnológica como mecanismo para impulsar el crecimiento económico Evidencia regional para México. Contaduría y Administración, 58(3), 11-37.

32. Rodeiro-Pazos, D. \& Calvo-Babio, N. (2012). El rol de los parques científico-tecnológicos en el emprendimiento universitario. Propuesta de un catálogo de indicadores de evaluación. GCG. Revista De Globalización, Competitividad y Gobernabilidad, 6(2), 95-109.

33. Rodríguez, J. F., Hernández Vasconcelos, M. A. \& Martínez Pérez, L. (2017). Estudio de percepción del potencial de las empresas de base tecnológica (startups) para el desarrollo y crecimiento económico de Tabasco, México. Investigación Operacional, 38(5), 455-461.

34. Romo, Vázquez, H. \& Rivas Tovar, L.A. (2012). Modelo de competitividad de las empresas operadoras de telefonía móvil en México. Contaduría y Administración, 57(1) enero-marzo 2012, 123148.

35. Santillán, R., Gaona, E. \& Hernández, N. (2015). El perfil del emprendedor que apoyan los fondos de capital privado/capital emprendedor en México. Contaduría y Administración, 60(1), 149174.

36. Urueña-López, A. \& Hidalgo-Nuchera, A. (2013). La información en la economía del conocimiento: retos y oportunidades para España. $E l$ Profesional de la Información, 22(4), 339-345. 